\title{
Far-field subdiffraction optical microscopy using metamaterial crystals: Theory and simulations
}

\author{
Alessandro Salandrino and Nader Engheta* \\ Department of Electrical and Systems Engineering, University of Pennsylvania, Philadelphia, Pennsylvania 19104, USA
}

(Received 26 May 2006; published 15 August 2006)

\begin{abstract}
Here we suggest and explore theoretically an idea for a far-field scanless optical microscopy with a subdiffraction resolution. We exploit the special dispersion characteristics of an anisotropic metamaterial crystal that is obliquely cut at its output plane, or has a curved output surface, in order to map the input field distribution onto the crystal's output surface with a compressed angular spectrum, resulting in a "magnified" image. This can provide a far-field imaging system with a resolution beyond the diffraction limits while no scanning is needed.
\end{abstract}

DOI: 10.1103/PhysRevB.74.075103

PACS number(s): 78.67.Pt, 07.60.Pb, 42.79.-e, 78.20.-e

\section{INTRODUCTION}

The microscope is one of the important instruments of scientific research in a variety of fields, from biology to surface science, to medicine. Evidently, we want to "see" what we study. For this reason any new imaging technique, or any improvement to the existing ones, is always welcome by the scientific community. ${ }^{1-3}$ For long it has been believed that the resolution of an optical instrument was limited by the operating wavelength of the light. Such resolution limitations of conventional far-field optics are indeed well known and they are due to the wave nature of light, which prevents it from being focused beyond the so-called Abbe-Rayleigh limit. ${ }^{4,5}$ This is imposed by the physics of the electromagnetic wave propagation in a homogeneous isotropic medium and so it cannot be avoided by any means. Nonetheless, in the past decades new forms of microscopy have been devised to overcome the diffraction resolution limits. An idea by Synge in 1928 (Ref. 6) led eventually to the realization of the first near-field scanning optical microscope in $1972,{ }^{7}$ followed by various refinements and variations from the original technique. The common theme of these techniques has been based on collecting the field in very close proximity of the sample by scanning a fiber tip. These new techniques have resulted in much finer resolutions beyond the diffraction limitation for an optical instrument and have led to the possibility of resolving details on the $10-100 \mathrm{~nm}$ scale. ${ }^{8}$ Another approach to the subwavelength resolution is the method developed by Hell and his co-workers, ${ }^{9,10}$ in which stimulated emission is utilized in fluorescence microscopy.

One of the constraints of some of these techniques may be the need for scanning the sample point by point (or region by region), making the entire procedure relatively slow and thus, in principle, preventing it from capturing fast dynamical processes taking place in the sample in real time. In a far-field optical microscope, however, the light scattered by the sample may be collected by the instrument aperture all at once, making the procedure fast and thus providing the possibility of observing temporally dynamic samples (which may be of paramount importance in biology and medicine, for instance). But the "bottle-neck" issue remains as the resolution for such far-field imaging.

Is there any way to improve the resolution of a far-field optical system such that to allow resolution beyond the dif- fraction limit? A very interesting technique is devised by Smolyaninov et al. ${ }^{11}$ based on the high effective refractive index exhibited by a plasmon-polariton propagation along a metal-dielectric interface. In their method, magnification occurs due to the curved geometry of a glycerin drop acting as a "mirror" around the sample. The amount of such magnification in their technique depends on the shape of the glycerin, which may be different from case to case.

Here we present another approach based on the peculiar propagation characteristics of a properly designed layered uniaxial metamaterial or "crystal" that can lead to far-field optical microscopy of samples with details finer than the operating wavelength. We show, theoretically and using computer simulations, that under proper conditions an image of a sample on a plane inside such a crystal can be "transferred" to another plane inside the medium while the image remains, in principle, essentially undistorted, but "larger." With this approach the image is magnified in the near field of the sample before the effects of the diffraction and free-space propagation take place, thus allowing far-field reconstruction with fine details that we would otherwise lose without the presence of the crystal.

\section{GEOMETRY OF THE CRYSTAL AND ITS PROPAGATION CHARACTERISTICS}

In recent years, metamaterials have been the subject of intense interest in various science and engineering research communities (e.g., see Ref. 12). These engineered materials can be constructed to exhibit exciting properties in their interaction with electromagnetic and optical signals. One part of our proposed imaging system described here is based on a one-dimensionally periodic layered metamaterial slab, depicted in Fig. 1.

The unit cell of this periodic structure is formed by two thin slices with thicknesses $d_{1}$ and $d_{2}$, made of materials with differing relative permittivities $\varepsilon_{1}$ and $\varepsilon_{2}$. We denote $d \equiv d_{1}$ $+d_{2}$, and thus we use two coefficients $c_{1}$ and $c_{2}$ as $d_{1}=c_{1} d$ and $d_{2}=c_{2} d$, where dimensionless coefficients satisfy $c_{1}+c_{2}$ $=1$. In addition to $\varepsilon_{1}$ and $\varepsilon_{2}$, these two coefficients also offer degrees of freedom in this design. This structure is inherently anisotropic and, if the thickness of the unit cell $d$ is small enough with respect to the operating wavelength, it has been shown (e.g., in Refs. 13 and 14) that the crystal can be in- 


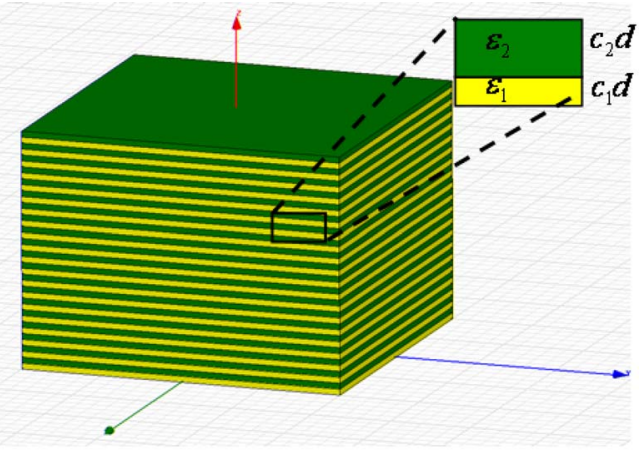

FIG. 1. (Color online) Structure of the metamaterial crystal. In the inset the various parameters are shown.

deed considered as an anisotropic metamaterial with a permittivity tensor given by

$$
\bar{\varepsilon}=\varepsilon_{0}\left(\begin{array}{ccc}
\varepsilon_{T} & 0 & 0 \\
0 & \varepsilon_{T} & 0 \\
0 & 0 & \varepsilon_{z}
\end{array}\right)
$$

with $\varepsilon_{T}=\left(c_{1} \varepsilon_{1}+c_{2} \varepsilon_{2}\right) /\left(c_{1}+c_{2}\right)$ and $\varepsilon_{z}=\left(c_{1}+c_{2}\right) \varepsilon_{1} \varepsilon_{2} /$ $\left(c_{2} \varepsilon_{1}+c_{1} \varepsilon_{2}\right)$.

This structure has been studied by several groups for different purposes (see e.g., Refs. 14-17), where interesting electromagnetic properties have been outlined, and salient features in the case of $\operatorname{Sign}\left(\varepsilon_{z}\right) \neq \operatorname{Sign}\left(\varepsilon_{T}\right)$ have been investigated. ${ }^{14-17}$ With this condition, the permittivity tensor becomes similar to that of anisotropic plasma and thus provides similar propagation characteristics - a subject that has been extensively investigated in the past (see, for example Refs. 18 and 19). One of the most striking phenomena occurring in wave propagation in such media is the cones of resonance, ${ }^{20,21}$ studied extensively by Balmain et al. ${ }^{21}$ This can be derived from the dispersion relation $\left(k_{T}^{2} / \varepsilon_{z}\right)$ $+\left(k_{z}^{2} / \varepsilon_{T}\right)=k_{0}^{2}$ for a plane wave with wave vector $\mathbf{k}=\mathbf{k}_{T}+k_{z} \hat{\mathbf{z}}$ in such a media. ${ }^{13}$

In the $k$ space, this equation represents either an ellipse or a hyperbola depending on the signs of the permittivity tensor elements. When $\operatorname{sign}\left(\varepsilon_{z}\right) \neq \operatorname{sign}\left(\varepsilon_{T}\right)$, the dispersion relation represents a hyperbola with the asymptotes given by $k_{z}$ $= \pm \sqrt{\left|\varepsilon_{T} / \varepsilon_{z}\right|} k_{T}{ }^{14-17}$ Owing to the hyperbolic shape of the equifrequency curves, the high spatial frequency components of any field propagate closer and closer to the direction given by the asymptotes of this hyperbola in a raylike fashion. This is consistent with what has been shown for the anisotropic plasma studied by Balmain et al., ${ }^{21}$ where they considered two-dimensional arrays of lumped inductors and capacitors in the microwave frequencies. ${ }^{21}$ (In the optical regime, if these lumped inductors and capacitors are replaced with the nanoinductors and nanocapacitors using plasmonic and nonplasmonic nanoparticles described in, Ref. 22 in the limit of these nanoparticles being packed, one would obtain the metamaterial layered structures shown in Fig. 1.) If the parameters $c_{1}, c_{2}, \varepsilon_{1}$, and $\varepsilon_{2}$ are chosen such that $\varepsilon_{T} \rightarrow 0$, the "cone of resonance" collapses into a single ray, almost undistorted. ${ }^{15-17}$ In other words the field distribution in an

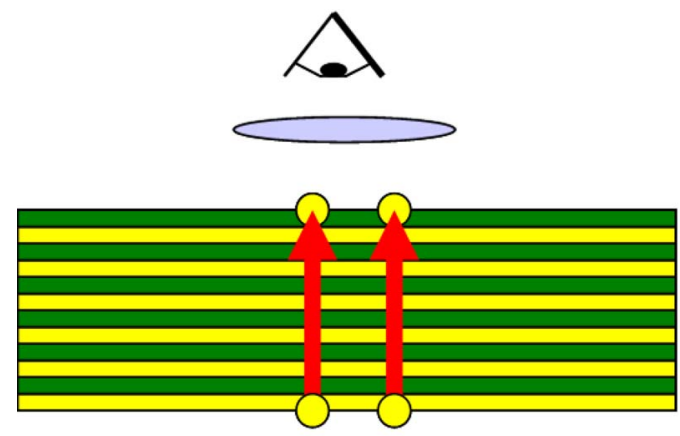

FIG. 2. (Color online) Sketch of the "nonmagnifying" setup.

arbitrary plane is transferred, point by point, by those parallel rays to any other arbitrary plane, in general, almost undistorted under the proper conditions on the permittivity tensor elements. This feature is in agreement with the general description and condition studied in Refs. 14-17. It is this $\varepsilon_{T}$ $\rightarrow 0$ condition that will be exploited in our proposed far-field imaging system, as described in the next section.

\section{THEORY OF FAR-FIELD SUBDIFFRACTION OPTICAL MICROSCOPY (FSOM)}

First, let us consider the above-mentioned metamaterial crystal with a finite number of layers, where the outer face of the first bottom layer is the input (i.e., object) plane of our imaging system while the external face of the last layer is the output plane, which may be viewed by a conventional farfield optical microscope, i.e., the microscope would be to focus on this output plane. If we have a certain field distribution on the input plane, under the condition $\varepsilon_{T} \rightarrow 0$ and no material loss, an exact copy of the input distribution will be transferred to the output plane through the set of rays parallel to the axis of stratification, as studied in Refs. 15-17. Although these "nondiffracting" rays are interesting by themselves, this setup, as shown in Fig. 2, with the input and the output planes in parallel does not help us to achieve our goal of far-field microscopy of the input distribution with subdiffraction resolution. This is because this has just created an exact replica of the original field of the input plane on the parallel output plane. In this case, assuming the relative permittivity of the outside medium to be $\varepsilon_{r}$, for the field distribution on the output plane, the components of the angular spectrum lying outside of the range $\left[-k_{0} \sqrt{\varepsilon_{r}}, k_{0} \sqrt{\varepsilon_{r}}\right]$ do not reach the far zone imager and/or detector, and therefore a conventional diffraction-limited image will be formed in the far zone. This implies that with this setup two radiating points (e.g., sources) on the input plane, separated by a distance shorter than $\lambda_{0} / 2 \sqrt{\varepsilon_{r}}$, will not be resolved in the far zone.

Is there any way to make those two sources appear to be farther apart on the output plane so that a far-field microscope can resolve them? To answer this question let us consider again another similar metamaterial crystal with an input and an output plane, but this time let us assume that the output plane is made with an oblique cut not parallel to the input face, as shown in Fig. 3. 


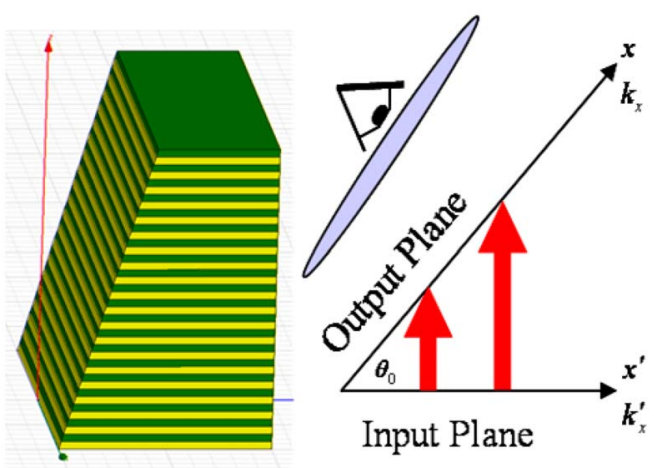

FIG. 3. (Color online) Sketch of the idea of the "magnifying setup" with an oblique cut.

For this case, if the distance between two sources on the input plane is $d^{\prime}$, under the previous condition $\varepsilon_{T} \rightarrow 0$ and lossless crystal, their images will be transferred to the oblique output plane by means of two parallel rays, and the image separation will appear to be $d=d^{\prime} / \cos \left(\theta_{0}\right)$ on that plane. Since $\cos \left(\theta_{0}\right)<1$, the "secondary sources" generated on the output plane can be far enough apart to be resolved by a conventional far-field optical microscope focused on the output plane. Here below we show this feature analytically, and provide design criteria for the system and discuss the limitations imposed by the use of realistic lossy materials.

Our analysis shows how a properly designed and obliquely cut metamaterial crystal can modify the angular spectrum of an input field distribution in order to allow a conventional far-field microscope to image the input spatial details with a resolution higher than $\lambda_{0} / 2 \sqrt{\varepsilon_{r}}$. For this, let us assume that an $x^{\prime}$-polarized TEM wave is incident normally on the input face of the crystal. The presence of an object on the input face of the crystal will result in a certain field distribution that we would like to image by this system. Using the notation sketched in Fig. 3, we can consider a magnetic field distribution $h_{y}\left(x^{\prime}\right)$ at the input plane whose angular spectrum is given by $\tilde{h}_{y}\left(k_{x}^{\prime}\right)$. Similarly we denote the field distribution and the angular spectrum at the output face as $H_{y}(x)$ and $\widetilde{H}_{y}\left(k_{x}\right)$, respectively. The field distribution at the output face can be written in terms of the angular spectrum on the input face using the following expression:

$$
H_{y}(x, 0)=\int_{-\infty}^{\infty} T\left(k_{x}^{\prime}\right) \tilde{h}_{y}\left(k_{x}^{\prime}\right) e^{i k_{x}^{\prime} x \cos \left(\theta_{0}\right)} e^{i \sqrt{k_{0}^{2} \varepsilon_{T}-k_{x}^{\prime 2} \varepsilon_{T} / \varepsilon_{z}} x \sin \left(\theta_{0}\right)} d k_{x}^{\prime},
$$

where $T\left(k_{x}^{\prime}\right)$ represents the transmission coefficient at the output face.

Under the condition $\varepsilon_{T}=0$, our analysis has shown that the transmission coefficient is unity and the second exponential term in Eq. (2) also becomes unity. The angular spectrum at the output face can then be easily shown to be

$$
\begin{aligned}
\tilde{H}_{y}\left(k_{x}\right) & =\int_{-\infty}^{\infty} \tilde{h}_{y}\left(k_{x}^{\prime}\right) \delta\left[k_{x}-k_{x}^{\prime} \cos \left(\theta_{0}\right)\right] d k_{x}^{\prime} \\
& =\frac{1}{\cos \left(\theta_{0}\right)} \tilde{h}_{y}\left[\frac{k_{x}}{\cos \left(\theta_{0}\right)}\right] .
\end{aligned}
$$

This demonstrates that this crystal, under the condition $\varepsilon_{T}$ $=0$ and lossless materials, transfers the field from the input plane to the oblique output plane, in principle, without any distortion, compressing the angular spectrum by a factor of $\cos \left(\theta_{0}\right)$ or, in other words, enlarging the image by a factor of $\cos \left(\theta_{0}\right)^{-1}$ along one of the $\mathrm{x}$ axis. Magnification along the $\mathrm{y}$ axis can be achieved by having the output plane of one crystal as the input plane of a second crystal with a different output cut.

Ideally, in order to meet the condition $\varepsilon_{T}=0$, one would like to utilize lossless materials with relative permittivities related by

$$
\varepsilon_{2}=-\left(c_{1} / c_{2}\right) \varepsilon_{1} .
$$

In other words one of the materials has to have a permittivity with a negative real part, which can be found, for instance, in many noble metals (e.g., Ag and $\mathrm{Au}$ ) as plasmonic media below their plasma frequency. ${ }^{23}$ However, due to material losses, the ideal condition $\varepsilon_{T}=0$ can only be approximately met. This in turn leads to a broadening and attenuation of the rays propagating from the input to the output planes in the crystal. While satisfying the condition (4), this effect can in part be reduced by decreasing the thickness of the layers made of the material with higher losses. As can be intuitively predicted, the rays that travel a longer distance are more attenuated, but this effect can, in principle, be compensated through a nonuniform illumination of the input plane. Here in Fig. 4, we present two-dimensional (2D) finite-elementmethod (FEM) simulations (using the software package COMSOL Multiphysics ${ }^{\mathrm{TM}}$ ), comparing the ideal lossless structure (as an effective medium) with a "realistic" structure (with some loss included) (as an effective medium), where the plasmonic material is considered to be silver illuminated at $633 \mathrm{~nm}(\varepsilon=-18+i 0.5)$ and the dielectric is glass $(\varepsilon$ $=2.2$ ). In these $2 \mathrm{D}$ simulations, we used the anisotropic crystal with the effective permittivity tensor given in Eq. (1) with $\varepsilon_{1}=-18+i 0.5, \varepsilon_{2}=2.2, c_{1}=0.11$, and $c_{2}=0.89$ and in the lossy case the two sources are illuminated unequally, with an amplitude ratio of $1: 2.1$, in order to compensate for the attenuation the longer ray suffers. The broadening of the rays in the lossy case is due to the opening of the cone of resonance when the condition (4) is not exactly fulfilled. In such cases, the system may be made more robust by making $\varepsilon_{z}$ large with choosing materials with oppositely signed, but nearly equal magnitude, permittivities. Alternatively, to reduce the effect of loss in negative-epsilon layers and thus keeping the rays sharper, one may consider some optical gain in the positive-epsilon layers, as suggested in Ref. 25. Even with the broadening of the rays, the magnification at the output face is still present. The intensity distribution on the output face can then be detected in far field through a conventional optical microscope with a focal plane at the output face. Subdiffraction resolution is therefore achieved by gen- 

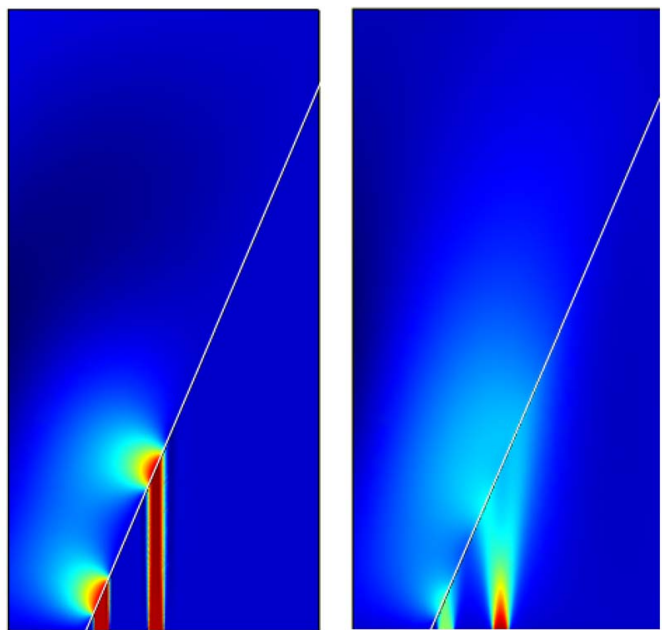

FIG. 4. (Color online) Finite-element-method simulations of a 2D lossless (left panel) and a lossy (right panel) anisotropic crystal with an oblique cut are shown. The distribution of the magnitude of the complex magnetic field of a 2D transverse magnetic (TM) excitation is shown here. The parameters of the anisotropic medium are given in the text. The simulated two "sources" at the input plane, and their images at the output plane, which are more separated, are clearly seen. The effects of the material loss in broadening and in attenuation of the rays are evident in the right panel, where the ratio of the source amplitudes is 1:2.1.

erating a preenlarged image of the input distribution on the output face of the crystal, followed by a far-field imaging system. The problem of the different attenuation experienced by beams traveling different distances may also be avoided by using a curved geometry such as spherical geometries (or 2D cylindrical geometries as shown in Figs. 5 and 6) of thin layers of materials with $\varepsilon_{1}$ and $\varepsilon_{2}$, which has also the advan-

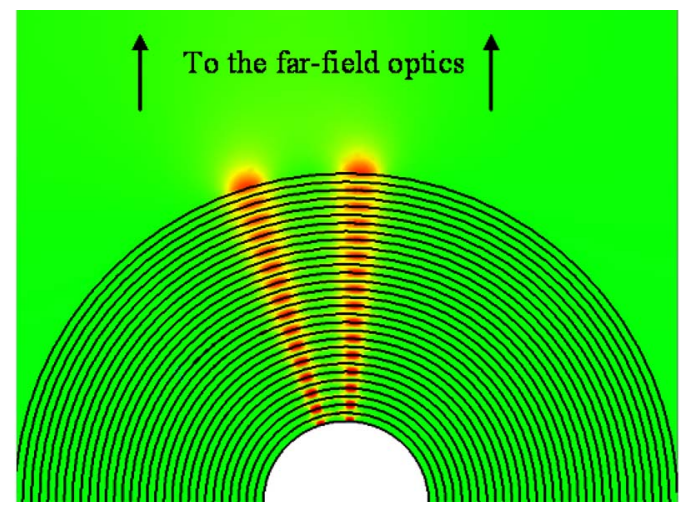

FIG. 5. (Color online) Finite-element-method simulation of 2D cylindrical geometry, with two sources with subwavelength separation. The distribution of the magnitude of the complex magnetic field of a 2D transverse magnetic (TM) excitation is shown. Here, to show the principle of operation of the curved structure, we have an ideal lossless configuration where the permittivities of the layers are 2 and -2 . With a proper design of these layers, the "spots" at the output surface can be separated with a distance larger than a necessary resolution of a conventional optical microscope. Thus, the entire system can act as a scanless optical microscope with beyonddiffraction-limit resolution.
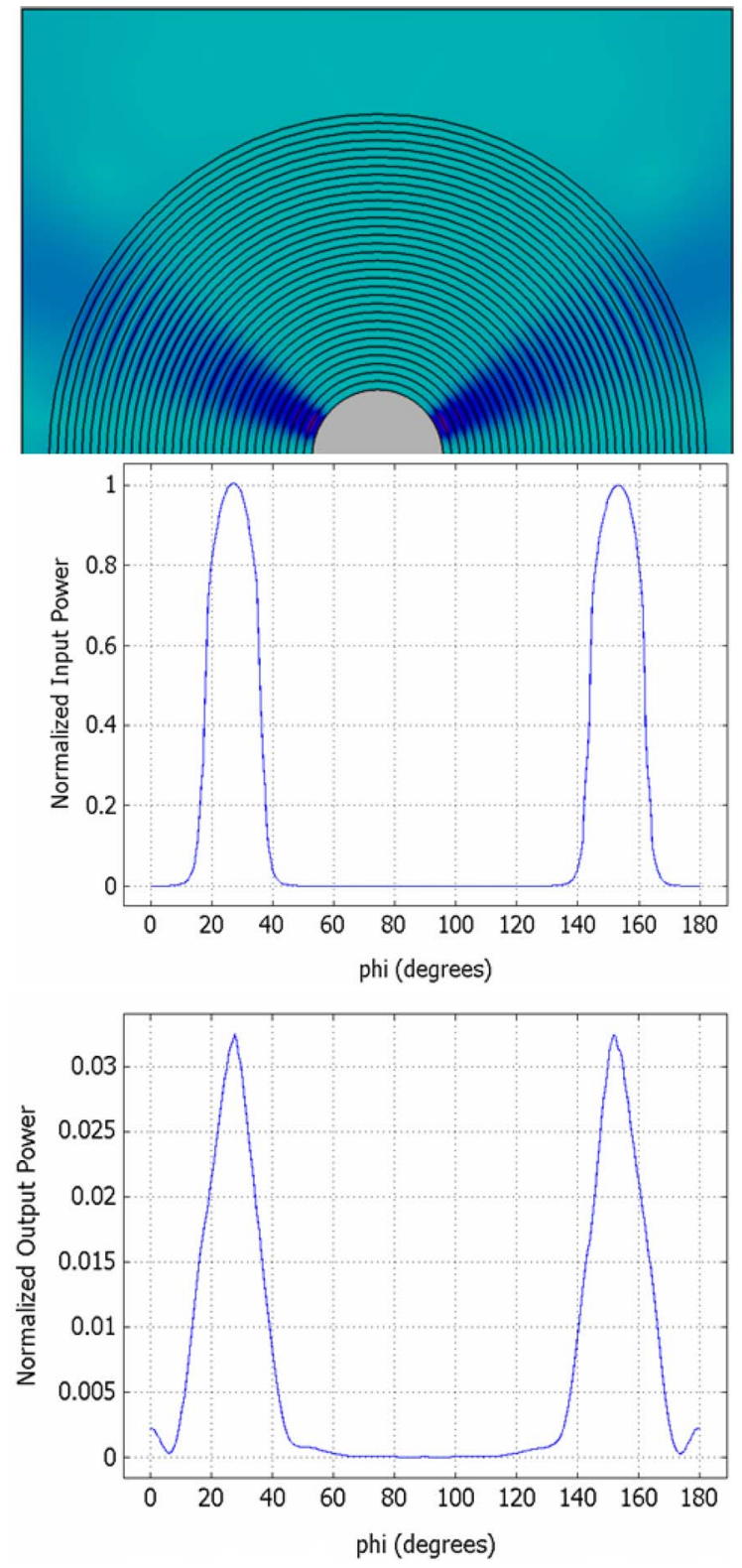

FIG. 6. (Color online) Finite-element-method simulation of a 2D cylindrical layered structure with some realistic materials for these layers. The distribution of the magnitude of the complex magnetic field of a 2D transverse magnetic (TM) excitation is shown in the top panel. The operating wavelength is assumed to be $410 \mathrm{~nm}$. The inner radius is $\lambda / 4$ and the radius of the output face is $5 \lambda / 4$. The thickness of the layers is $\lambda / 32$. The materials used for layers are silver (dielectric constant $-5.08+i 0.226$ at $410 \mathrm{~nm}$ ) and diamond (dielectric constant 5.08). The middle panel shows the normalized input power as a function of the angle at the input face. The bottom panel shows the output power as a function of the angle at the output face. The structure shows a magnification of a factor 5 , therefore allowing us to resolve in far zone details down to $\lambda / 10$.

tage of providing local magnification. In the case of cylindrical (spherical) geometries the angular distribution of field along $\varphi($ and $\theta)$ is preserved, so that the magnification occurs as the radius of curvature increases from the input face to the output face: the image turns out to be enlarged by a factor $r_{\text {out }} / r_{\text {in }}$, where $r_{\text {out }}$ and $r_{\text {in }}$ are the radii of the output and of 
the input face, respectively [it must be noted that this kind of magnification is different from that suggested in Ref. 24 where the "growth" of the evanescent field in the near field is involved, whereas in the present method the special dispersion of anisotropic crystal (i.e., as an effective medium) and its curvature provide such magnification. Moreover in Ref. 24, the metamaterial layer is radially inhomogeneous, whereas here the radial layers are all alternately similar]. In this case then a near-field premagnification will allow for far-field imaging of details on the order of $\left(r_{\text {in }} / r_{\text {out }}\right) \lambda / 2$.

\section{CONCLUSIONS}

In this paper, we presented and studied theoretically a different far-field imaging system that allows for a resolution below the diffraction limit. The idea is based on the peculiar dispersion characteristics of an obliquely cut (or curved output) anisotropic metamaterial with $\varepsilon_{T} \rightarrow 0$ in order to project an input field distribution on an oblique plane (or curved output plane) with magnified angular spectrum. This provides a magnification, before conventional optical instruments are used in far fields to detect the image, thus resulting in the possibility of achieving subwavelength resolution using far-field microscopy.

\section{ACKNOWLEDGMENT}

This work was supported in part by the U.S. Air Force Office of Scientific Research (AFOSR) Grant No. FA955005-1-0442.
*To whom correspondence should be addressed. Email address: engheta@ee.upenn.edu

${ }^{1}$ F. Zernike, http://nobelprize.org/physics/laureates/1953/ (1953).

${ }^{2}$ E. Ruska, http://nobelprize.org/physics/laureates/1986/ (1986).

${ }^{3}$ G. Binnig and H. Rohrer, http://nobelprize.org/physics/laureates/ 1986/ (1986).

${ }^{4}$ E. Abbe, Schultzes Arch. Mikr. Anat. 9, 413 (1873).

${ }^{5} \mathrm{M}$. Born and E. Wolf, Principles of Optics, 7th ed. (Cambridge University Press, Cambridge, 1999).

${ }^{6}$ E. H. Synge, Philos. Mag. 6, 356 (1928).

${ }^{7}$ E. A. Ash and G. Nicholls, Nature (London) 237, 510 (1972).

${ }^{8}$ J. Koglin, U. C. Fischer, and H. Fuchs, Phys. Rev. B 55, 7977 (1997).

${ }^{9}$ S. W. Hell and J. Wichmann, Opt. Lett. 19, 780 (1994).

${ }^{10}$ T. A. Klar et al., Proc. Natl. Acad. Sci. U.S.A. 97, 8206 (2000).

${ }^{11}$ I. I. Smolyaninov, J. Elliott, A. V. Zayats, and C. C. Davis, Phys. Rev. Lett. 94, 057401 (2005).

${ }^{12}$ N. Engheta and R. W. Ziolkowski, IEEE Trans. Microwave Theory Tech. 53, 1535 (2005).

${ }^{13}$ S. Tretyakov, Analytical Modeling in Applied Electromagnetics
(Artech House, Norwood, MA, 2000).

${ }^{14}$ S. Anantha Ramakrishna, J. B. Pendry, M. C. K. Wiltshire, and W. J. Stewart, J. Mod. Opt. 50, 1419 (2003).

${ }^{15}$ S. Feng and J. Elson, Opt. Express 14, 216 (2006).

${ }^{16}$ D. Schurig and D. R. Smith, New J. Phys. 7, 162 (2005).

${ }^{17}$ P. A. Belov and Y. Hao, Phys. Rev. B 73, 113110 (2006).

${ }^{18}$ C. H. Papas, Theory of Electromagnetic Wave Propagation (Dover Publications, New York, 1988).

${ }^{19}$ L. B. Felsen and N. Marcuvitz, Radiation and Scattering of Waves (IEEE Press, Piscataway, NJ, 1994).

${ }^{20}$ R. K. Fisher and R. W. Gould, Phys. Rev. Lett. 22, 1093 (1969).

${ }^{21}$ K. G. Balmain, A. A. E. Luttgen, and P. C. Kremer, IEEE Antennas Wireless Propag. Lett. 1, 146 (2002).

${ }^{22}$ N. Engheta, A. Salandrino, and A. Alù, Phys. Rev. Lett. 95, 095504 (2005).

${ }^{23}$ E. D. Palik, Handbook of Optical Constants of Solids (Academic Press, San Diego, CA, 1991).

${ }^{24}$ J. B. Pendry, Opt. Express 11, 755 (2003).

${ }^{25}$ S. A. Ramakrishna and J. B. Pendry, Phys. Rev. B 67, 201101(R) (2003). 